\title{
ABO Blood Groups and the Incidence of Complications in COVID-19 Patients: A Population-Based Prospective Cohort Study
}

\author{
Salvador Domènech-Montoliu ${ }^{1}$, Joan Puig-Barberà ${ }^{2} \oplus$, Maria Rosario Pac-Sa ${ }^{3}$, Paula Vidal-Utrillas ${ }^{4}$, \\ Marta Latorre-Poveda ${ }^{1}$, Alba Del Rio-González ${ }^{4}$, Sara Ferrando-Rubert ${ }^{4}$, Gema Ferrer-Abad ${ }^{4}$, \\ Manuel Sánchez-Urbano ${ }^{1}$, Laura Aparisi-Esteve ${ }^{5}{ }^{(0)}$, Gema Badenes-Marques ${ }^{1}$, Belén Cervera-Ferrer ${ }^{1}$, \\ Ursula Clerig-Arnau ${ }^{1}$, Claudia Dols-Bernad ${ }^{6}$, Maria Fontal-Carcel ${ }^{7}$, Lorna Gomez-Lanas ${ }^{1}$, David Jovani-Sales ${ }^{1}{ }^{1}$, \\ Maria Carmen León-Domingo ${ }^{8}$, Maria Dolores Llopico-Vilanova ${ }^{1}$, Mercedes Moros-Blasco ${ }^{5}$, \\ Cristina Notari-Rodríguez ${ }^{6}$, Raquel Ruíz-Puig ${ }^{1}$, Sonia Valls-López ${ }^{1}$ and Alberto Arnedo-Pena ${ }^{3,9,10, *(D)}$
}

check for

updates

Citation: Domènech-Montoliu, S.;

Puig-Barberà, J.; Pac-Sa, M.R.;

Vidal-Utrillas, P.; Latorre-Poveda, M.;

Rio-González, A.D.; Ferrando-Rubert,

S.; Ferrer-Abad, G.; Sánchez-Urbano,

M.; Aparisi-Esteve, L.; et al. ABO

Blood Groups and the Incidence of

Complications in COVID-19 Patients:

A Population-Based Prospective

Cohort Study. Int. J. Environ. Res.

Public Health 2021, 18, 10039. https://

doi.org/10.3390/ijerph181910039

Academic Editors: Hyeoneui Kim,

Jeeyae Choi, Albert Park and

Nansu Zong

Received: 27 July 2021

Accepted: 16 September 2021

Published: 24 September 2021

Publisher's Note: MDPI stays neutral with regard to jurisdictional claims in published maps and institutional affiliations.

Copyright: (c) 2021 by the authors. Licensee MDPI, Basel, Switzerland. This article is an open access article distributed under the terms and conditions of the Creative Commons Attribution (CC BY) license (https:// creativecommons.org/licenses/by/ $4.0 /)$.
1 Emergency Service Hospital de la Plana, Health Department 3, 12540 Vila-Real, Spain; pttcarmen@hotmail.com (S.D.-M.); martalapo@hotmail.com (M.L.-P.); manu.msu@gmail.com (M.S.-U.); gemabamar@hotmail.com (G.B.-M.); belencerveraferrer@hotmail.es (B.C.-F.); ursuclerig@gmail.com (U.C.-A.); lornag178@gmail.com (L.G.-L.); jovasal1987@gmail.com (D.J.-S.); 1lopivila@hotmail.com (M.D.L.-V.); raquelruizpuig@gmail.com (R.R.-P.); Sonia.valls.lopez@gmail.com (S.V.-L.)

2 Vaccines Research Area FISABIO, 46020 Valencia, Spain; jpuigb55@gmail.com

3 Public Health Center, Health Department 2, 12003 Castello de la Plana, Spain; charopac@gmail.com

4 Health Centers I and II, Health Department 2, 12530 Borriana, Spain; vidalutrillaspaula@gmail.com (P.V.-U.); delrio_alb@gva.es (A.D.R.-G.); sfr1812@gmail.com (S.F.-R.); gferrer@uji.es (G.F.-A.)

5 Carinyena Health Center, 12540 Vila-Real, Spain; lauraaparisiesteve@gmail.com (L.A.-E.); mercedesmb1094@gmail.com (M.M.-B.)

6 Health Center, Health Department 3, 12200 Onda, Spain; claudiadb1294@hotmail.com (C.D.-B.); notari_cri@gva.es (C.N.-R.)

7 Health Center, Health Department 3, 12600 La Vall d'Uixó, Spain; fontalcarcel93@hotmail.com

8 Villa Fátima School, Health Department 3, 12530 Borriana, Spain; carmendole@hotmail.com

9 Department of Health Science, Public University Navarra, 31008 Pamplona, Spain

10 Epidemiology and Public Health (CIBERESP), 28029 Madrid, Spain

* Correspondence: albertoarnedopena@gmail.com or arnedo_alb@gva.es; Tel.: +35-622-573979

\begin{abstract}
After a COVID-19 outbreak in the Falles festival of Borriana (Spain) during March 2020, a cohort of patients were followed until October 2020 to estimate complications post-COVID-19, considering ABO blood groups (ABO). From 536 laboratory-confirmed cases, 483 completed the study $(90.1 \%)$ carried by the Public Health Center of Castello and the Emergency and Microbiology and Clinical Analysis of Hospital de la Plana Vila-real. The study included ABO determination and telephone interviews of patients. The participants had a mean age of $37.2 \pm 17.1$ years, 300 females $(62.1 \%)$. ABO were $\mathrm{O}(41.4 \%), \mathrm{A}(45.5 \%), \mathrm{B}(9.1 \%)$, and $\mathrm{AB}(3.9 \%)$. We found no difference in the incidence of COVID-19 infections. A total of 159 (32.9\%) patients reported one or more post-COVID19 complications with divergent incidences after adjustment: $\mathrm{O}(32.3 \%), \mathrm{A}(32.6 \%), \mathrm{B}(54.1 \%)$, and $\mathrm{AB}$ (27.6\%); B groups had more complications post-COVID-19 when compared with O group (adjusted relative risk [aRR] 95\% confidence interval [CI] 1.68, 95\% CI 1.24-2.27), and symptoms of fatigue (1.79, 95\% CI 1.08-2.95), myalgia (2.06, 95\% CI 1.10-3.84), headache (2.61, 95\% CI 1.58-4.31), and disorder of vision (4.26 95\% CI 1.33-13.60). In conclusion, we observed significant differences in post-COVID-19 complications by ABO, with a higher incidence in B group. Additional research is justified to confirm our results.
\end{abstract}

Keywords: COVID-19; post-COVID-19; complications; symptoms; ABO blood groups; incidence; cohort; population based 


\section{Introduction}

Ongoing symptomatology in COVID-19 patients after the acute phase of the illness is frequent. It has been characterized as a syndrome of long or persistent COVID, affecting both children and adults [1-3]. The research on the risk and protective factors of infection and severity of COVID-19 is crucial in the second year of the pandemic. Thus far, many potential factors have been considered $[4,5]$.

Among these factors, the $\mathrm{ABO}$ blood groups $(\mathrm{ABO})$ are being studied with intensity [6-9], and it has been recommended to determine the ABO of COVID-19 patients to improve medical care [10].

There are, however, conflicting results about the role of $\mathrm{ABO}$ and the risk of COVID-19 disease. Some studies found the A and B blood groups related to a higher risk of infection, complications, and mortality [11-15]. In contrast, other studies have not found differences in disease severity among the $\mathrm{O}$ and A groups [16], whereas other studies have found the $\mathrm{O}$ group as protective, compared to the non-O blood groups. Finally, large and well-designed studies have not found that the ABO blood groups are a risk factor for COVID-19 infection or severity $[17,18]$.

Several conditions could explain this situation of conflicting results, including diversity of sample size, effect size, multiple confounders, publication bias, or even chance distributions $[17,19,20]$. Complications considering ABO and the follow-up of patients with a mild illness have been less studied [21-24].

This study aimed to estimate the frequency of persistent symptoms and complications in COVID-19 patients and its associations with $\mathrm{ABO}$ blood groups.

\section{Materials and Methods}

After a COVID-19 outbreak in the Falles festival with several mass gathering events (MGEs) in Borriana (Spain) during March 2020 [25], we followed a population-based prospective cohort of patients until October 2020 [26] to estimate disease evolution and incidence of complications post-COVID-19, and its relationship with participants ABO blood group. The study was carried by the Public Health Center of Castelló, and the Emergency and Microbiology and Clinical Analysis Services of Hospital de la Plana Vila-real (Spain).

We performed the first study of the COVID-19 outbreak during May-June 2020 through a serological survey and questionnaire interview [25]; we included 1338 people in the study, and we found 570 COVID-19 patients with 536 patients, laboratory-confirmed tests: electrochemiluminescence immunoassay (ECLIA) (Elecsys ${ }^{\circledR}$, Mannheim, Germany, Anti-SARS-CoV-2, Roche Diagnostics) [27], in 514 patients, lateral flow immunochromatographic assay (LFIC), in 15 patients, and reverse transcriptase-polymerase chain reaction (RT-PCR) in 39 patients [25]. ABO was determined by the gel test (ID-Card ABO/RhD, DiaMed GmbH, Bio-Rad Laboratories Switzerland) [28].

In October 2020, health staff of the Hospital de la Plana Vila-real, and health centers of Borriana, Vila-real, Onda, and La Vall d'Uixo conducted a telephone interview of each participant to obtain information about their health situation, medical assistance, illness's evolution, symptoms post-COVID-19, and duration. In addition, we acquired data from the May-June questionnaire on age, sex, weight, height, body mass index (BMI) $\left(\mathrm{kg} / \mathrm{m}^{2}\right)$, occupation, level of physical exercise, smoking habits, consumption of alcohol, chronic illness, and COVID-19 exposures. COVID-19 exposition included the following features: see a person with a cough at MGEs, attendance MGEs $\geq 2$, contact with a COVID-19 case, and family with COVID-19 case. 


\section{Statistical Methods}

The expected incidence of COVID-19 infection by the $\mathrm{ABO}$ was estimated from the distribution of $\mathrm{ABO}$ in three sources: two blood donor studies of Catalonia and Navarra $[13,29]$ and one study of an active general population of the Spanish Mediterranean zone [30]. We compared the observed $\mathrm{ABO}$ distribution with the expected distribution through Fisher's exact test. Distributions of $\mathrm{ABO}$ from the three sources were applied to the 1338 participants in the COVID-19 outbreak study [25] to obtain an incidence rate by each $\mathrm{ABO}$. We used the O group as the reference to estimate relative risks (RR) and 95\% confidence interval (CI) by Poisson regression.

Reported complications and symptoms were the dependent variables, and $\mathrm{ABO}$ was the predictive variable. We used the Chi2, Fisher's exact test, Wilcoxon matched-pairs signed-ranks test, and Kruskal-Wallis tests for unadjusted comparisons. After a review of medical literature, we used directed acyclic graphs [31], and identified age, sex, lifestyle, and COVID-19 exposures as confounding factors [32], and adjusted our models accordingly using inverse probability weight regression [33]. We performed all calculations with the statistical program STATA ${ }^{\circledR}$ version 14.

Following up this cohort was part of the public health surveillance as a prolongation of the COVID-19 outbreak control measures [25] and the response of the COVID-19 pandemic. It was exempted from Ethics Review Board approval's protocol according to the Spanish legislation. The study was approved by the director of the Public Health Center of Castelló and the management of the Health Department of La Plana. All participants or the parents of the minors provided their informed written consent to be included in the study.

\section{Results}

The participation rate was $90.1 \%(483 / 536)$ considering the patients with laboratoryconfirmed COVID-19 in the COVID-19 outbreak. The mean age was $37.2 \pm 17.1$ years (rank $1-81)$, with 300 females $(62.1 \%)$ and 183 males $(37.9 \%)$. ABO blood groups distribution was $\mathrm{O}(41.4 \%), \mathrm{A}(45.5 \%), \mathrm{B}(9.1 \%)$, and $\mathrm{AB}(3.9 \%)$. The subjects' characteristics by $\mathrm{ABO}$ are shown in Table 1 . We found no appreciable differences among $\mathrm{ABO}$ by demographic, lifestyle, and COVID-19 exposure.

Table 1. Distribution of characteristics ABO blood groups in the subjects included October 2020. Borriana COVID-19 cohort 2020.

\begin{tabular}{|c|c|c|c|c|c|}
\hline \multirow[t]{2}{*}{ Variables } & \multicolumn{4}{|c|}{ ABO Blood Groups $n=483$} & \multirow[t]{2}{*}{$p$-Value } \\
\hline & $\begin{array}{c}\mathrm{O} \\
N=200 \\
N(\%)\end{array}$ & $\begin{array}{c}\mathrm{A} \\
N=220 \\
N(\%)\end{array}$ & $\begin{array}{c}\text { B } \\
N=44 \\
N(\%)\end{array}$ & $\begin{array}{c}\mathrm{AB} \\
N=19 \\
N(\%)\end{array}$ & \\
\hline Female Sex & $127(63.5)$ & $131(59.5)$ & $31(70.5)$ & $11(57.9)$ & 0.528 \\
\hline Age (years) mean \pm standard desviation & $37.3 \pm 15.5$ & $37.4 \pm 16.9$ & $37.9 \pm 18.0$ & $33.7 \pm 13.5$ & 0.528 \\
\hline $0-14$ & $23(11.5)$ & $24(10.9)$ & $5(11.4)$ & $1(5.3)$ & \\
\hline $15-24$ & $39(19.5)$ & $36(16.4)$ & $9(20.5)$ & $5(26.3)$ & \\
\hline $25-34$ & $21(10.5)$ & $34(15.5)$ & $3(6.8)$ & $2(10.5)$ & \\
\hline $35-44$ & $40(20.0)$ & $39(17.7)$ & $9(20.5)$ & $9(47.4)$ & \\
\hline $45-54$ & $40(20.0)$ & $53(24.1)$ & $11(25.0)$ & $2(10.5)$ & \\
\hline $55-64$ & $28(14.0)$ & $27(12.3)$ & $6(13.6)$ & $0(0)$ & \\
\hline 65 and over & $9(4.5)$ & $7(3.2)$ & $1(2.3)$ & $0(0)$ & \\
\hline \multicolumn{6}{|l|}{ Occupation 1,2} \\
\hline Occupation I-II & $60(30.3)$ & $63(28.6)$ & $17(39.5)$ & $4(21.1)$ & 0.444 \\
\hline Occupation III-VI & $138(69.7)$ & $157(71.4)$ & $26(60.5)$ & $15(78.9)$ & \\
\hline Physical exercise & $115(57.5)$ & $135(61.4)$ & $29(67.4)$ & $10(52.6)$ & 0.656 \\
\hline Alcohol consumption ${ }^{3}$ & $44(23.0)$ & $45(20.8)$ & $12(27.9)$ & $7(36.8)$ & 0.336 \\
\hline \multicolumn{6}{|l|}{ Smoking 4} \\
\hline No smoking & $119(62.6)$ & $135(62.8)$ & $26(60.5)$ & $16(84.2)$ & 0.567 \\
\hline Ex smoking & $42(22.1)$ & $52(24.2)$ & $11(25.6)$ & $1(5.3)$ & \\
\hline Current smoker & $29(15.3)$ & $28(13.0)$ & $6(13.9)$ & $2(10.5)$ & \\
\hline
\end{tabular}


Table 1. Cont.

\begin{tabular}{|c|c|c|c|c|c|}
\hline \multirow[t]{2}{*}{ Variables } & \multicolumn{4}{|c|}{ ABO Blood Groups $n=483$} & \multirow[t]{2}{*}{$p$-Value } \\
\hline & $\begin{array}{c}\mathrm{O} \\
N=200 \\
N(\%)\end{array}$ & $\begin{array}{c}\mathrm{A} \\
N=220 \\
N(\%)\end{array}$ & $\begin{array}{c}\text { B } \\
N=44 \\
N(\%)\end{array}$ & $\begin{array}{c}\mathrm{AB} \\
N=19 \\
N(\%)\end{array}$ & \\
\hline \multicolumn{6}{|l|}{ Body Mass Index $\left(\mathrm{Kg} / \mathrm{m}^{2}\right)^{5}$} \\
\hline$<18.5$ & $20(10.1)$ & $15(6.9)$ & $5(11.6)$ & $1(5.3)$ & 0.321 \\
\hline $18.5-24.9$ & $92(46.2)$ & $86(39.6)$ & $20(46.5)$ & $11(57.9)$ & \\
\hline $25.0-29.9$ & $51(25.6)$ & $80(36.9)$ & $11(25.6)$ & $6(31.6)$ & \\
\hline$\geq 30.0$ & $36(18.1)$ & $36(16.6)$ & $7(16.3)$ & $1(5.3)$ & \\
\hline Chronic illness ${ }^{6}$ & $66(33.2)$ & $81(37.3)$ & $14(31.8)$ & $5(26.3)$ & 0.698 \\
\hline \multicolumn{6}{|l|}{ Exposure COVID-19 } \\
\hline See a person with a cough at mass gathering events ${ }^{7}$ & $91(45.7)$ & $93(43.1)$ & $15(34.9)$ & $6(31.6)$ & 0.442 \\
\hline Attendance mass gathering events $\geq 2$ & $121(60.5)$ & $135(61.4)$ & $27(61.4)$ & $11(57.9)$ & 0.989 \\
\hline Contact COVID-19 case ${ }^{8}$ & $161(81.7)$ & $179(82.5)$ & $35(81.4)$ & $14(73.7)$ & 0.774 \\
\hline Family COVID-19 case $^{9}$ & $127(63.5)$ & $131(59.5)$ & $32(74.4)$ & $13(68.4)$ & 0.292 \\
\hline
\end{tabular}

${ }^{1}$ Missing information 3 participants; ${ }^{2}$ occupation groups I-II: professional, managerial, and technical occupations; groups III-VI: skilled, non-manual or manual, partly skilled, unskilled occupations; ${ }^{3}$ missing information 14 participants; ${ }^{4}$ missing information 16 participants: ${ }^{5}$ missing information 5 participants; ${ }^{6}$ missing information 4 participants; ${ }^{7}$ missing information 6 participants; ${ }^{8}$ missing information 7 participants; ${ }^{9}$ missing information 1 participant.

We found no difference in the incidence of SARS-CoV-2 when comparing the observed proportion of $\mathrm{ABO}$ and the expected proportion from blood donors of Catalonia $(p=0.247)$ and Navarra $(p=0.089)$, and an active general population $(p=0.366)$ (Table 2). However, $\mathrm{RRs}$ of no-O groups presented a higher incidence than the estimated $\mathrm{O}$ group with a range of 1.06-2.06 and was significant when applying the two blood donor sources to the participants in the COVID-19 outbreak but not when using the active general population.

Symptoms and duration of the COVID-19 illness among ABO are shown in Table 3. $B$ group experienced a higher proportion of symptomatic disease, medical consultation, hospitalization, and a longer duration of illness. B group reported a higher presence of symptoms such as diarrhea, vomits, weakness, headache, and myalgia. Fever and loss of smell/taste presented significant differences with the other blood groups, $(p=0.020)$ and $(p=0.016)$, respectively. There was no difference in habitual health status before and after the COVID-19 considering the ABO blood group. We found significant differences in the $\mathrm{O}$ and $\mathrm{A}$ groups between the before and after the illness in health status $(p=0.000)$ and $(p=0.001)$; in contrast, little to no difference for the B group $(p=0.180)$ and AB group $(p=0.414)$. 


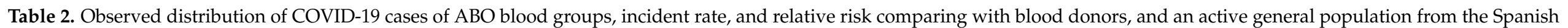
Mediterranean zone as the reference population. Borriana COVID-19 cohort 2020.

\begin{tabular}{|c|c|c|c|c|c|}
\hline \multirow[t]{2}{*}{ Variables } & \multicolumn{4}{|c|}{ ABO Blood Groups $n=483$} & \multirow[t]{2}{*}{$p$-Value } \\
\hline & $\begin{array}{c}\mathrm{O} \\
N(\%)\end{array}$ & $\begin{array}{c}\mathrm{A} \\
N(\%)\end{array}$ & $\begin{array}{c}\text { B } \\
N(\%)\end{array}$ & $\begin{array}{c}\mathrm{AB} \\
N(\%)\end{array}$ & \\
\hline $\begin{array}{l}\text { Observed distribution in the cohort } \\
\text { Blood donors } 1\end{array}$ & $200(41.41)$ & $220(45.55)$ & $44(9.11)$ & $19(3.93)$ & \\
\hline $\begin{array}{l}\text { Expected in reference population } \\
\text { COVID-19 Outbreak } n=1338\end{array}$ & $229(47.34)$ & $203(42.02)$ & $36(7.52)$ & $15(3.12)$ & 0.271 \\
\hline Expected in reference population & $633(47.34)$ & $562(42.02)$ & $101(7.52)$ & $42(3.12)$ & \\
\hline Incidence rate $\times 100$ & 31.6 & 39.1 & 43.6 & 45.2 & \\
\hline $\begin{array}{l}\text { Relative Risk 95\% CI }{ }^{2} \\
\text { Blood donors }{ }^{3}\end{array}$ & 1.00 & $1.24(1.02-1.50)$ & $1.37(0.99-1.91)$ & $1.43(0.89-2.29)$ & 0.008 \\
\hline $\begin{array}{l}\text { Expected in reference population } \\
\text { COVID-19 Outbreak } n=1338\end{array}$ & $232(48.03)$ & $208(42.99)$ & $31(6.43)$ & $12(2.55)$ & 0.089 \\
\hline Expected in reference population & $643(48.03)$ & $575(42.99)$ & $86(6.43)$ & $34(2.55)$ & \\
\hline Incidence rate $\times 100$ & 31.1 & 38.3 & 51.2 & 55.9 & \\
\hline Relative Risk 95\% CI ${ }^{2}$ & 1.00 & $1.23(1.02-1.49)$ & $1.64(1.19-2.28)$ & $1.80(1.12-2.88)$ & 0.000 \\
\hline $\begin{array}{c}\text { Active general population }{ }^{4} \\
\text { Expected in reference popopulation } \\
\text { COVID-19 Outbreak } n=1338\end{array}$ & $211(43.76)$ & $216(43.76)$ & $46(9.73)$ & $10(2.0)$ & 0.366 \\
\hline Incidence rate $\times 100$ & 34.2 & 36.8 & 34.4 & 70.4 & \\
\hline Relative Risk 95\% CI & 1.00 & $1.08(0.89-1.30)$ & $1.06(0.72-1.39)$ & $2.06(1.29-3.30)$ & 0.084 \\
\hline
\end{tabular}

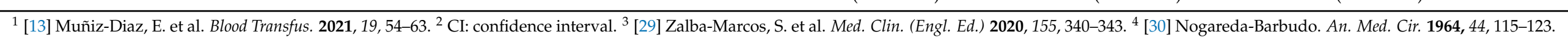


Table 3. Symptoms in the acute COVID-19 illness in March 2020 and previous health status and health status in October 2020 by ABO blood groups. Borriana COVID-19 cohort 2020.

\begin{tabular}{|c|c|c|c|c|c|}
\hline \multirow[t]{2}{*}{ Variables } & \multicolumn{4}{|c|}{ ABO Blood Groups $n=483$} & \multirow[t]{2}{*}{$p$-Value } \\
\hline & $\begin{array}{c}\mathrm{O} \\
N=200 \\
N(\%)\end{array}$ & $\begin{array}{c}\mathrm{A} \\
N=220 \\
N(\%)\end{array}$ & $\begin{array}{c}\text { B } \\
N=44 \\
N(\%)\end{array}$ & $\begin{array}{c}\mathrm{AB} \\
N=19 \\
N(\%) \\
\end{array}$ & \\
\hline Symptomatic & $178(89)$ & 193(87.7) & $41(93.2)$ & $17(89.5)$ & 0.804 \\
\hline Asymptomatic & $22(11)$ & $27(12.3)$ & $3(14.7)$ & $2(10.5)$ & \\
\hline Medical consultation for acute illness & $86(43)$ & $94(42.7)$ & $23(52.3)$ & $4(21.1)$ & 0.152 \\
\hline Hospitalization & $3(1.5)$ & $4(1.8)$ & $2(4.5)$ & $0(0)$ & 0.542 \\
\hline Illness duration ${ }^{1}$ & $11.7 \pm 13.4$ & $13.2 \pm 22.5$ & $13.7 \pm 14.9$ & $11.3 \pm 10.8$ & 0.911 \\
\hline \multicolumn{6}{|l|}{ Illness symptoms } \\
\hline Cough $^{2}$ & $85(42.5)$ & $93(42.3)$ & $16(36.4)$ & $8(42.1)$ & 0.899 \\
\hline Runny nose & $53(26.5)$ & $63(28.6)$ & $10(22.7)$ & $8(42.1)$ & 0.436 \\
\hline Throat pain & $56(28)$ & $66(30)$ & $16(36.4)$ & $7(36.8)$ & 0.618 \\
\hline Fever & $98(49)$ & $94(42.7)$ & $30(68.2)$ & $9(47.4)$ & 0.020 \\
\hline Loss smell/taste ${ }^{3}$ & $100(50)$ & $100(45.5)$ & $31(70.5)$ & $7(36.8)$ & 0.016 \\
\hline Diarrhea & $44(22)$ & $52(23.6)$ & $16(36.4)$ & $5(26.3)$ & 0.247 \\
\hline Vomits & $7(3.5)$ & $13(5.9)$ & $5(11.4)$ & $1(5.3)$ & 0.158 \\
\hline Weakness & $101(50.5)$ & $104(47.3)$ & $29(65.9)$ & $11(57.9)$ & 0.137 \\
\hline Headache & $76(38)$ & $93(42.3)$ & $23(52.3)$ & $7(36.8)$ & 0.379 \\
\hline Myalgia & $91(45.5)$ & $96(43.6)$ & $25(56.8)$ & $12(63.2)$ & 0.189 \\
\hline Dyspnea & $8(4)$ & $6(2.7)$ & $1(2.3)$ & $2(10.5)$ & 0.282 \\
\hline Skin's lesions 4 & $18(9)$ & $23(10.5)$ & $5(11.4)$ & $1(5.3)$ & 0.861 \\
\hline \multicolumn{6}{|l|}{ Habitual health status } \\
\hline Poor & $1(0.01)$ & $0(0)$ & $0(0)$ & $0(0)$ & 0.743 \\
\hline Fair & $7(3.5)$ & $8(3.6)$ & $4(9.1)$ & $0(0)$ & \\
\hline Good & $106(53)$ & $119(54.1)$ & $24(54.5)$ & $10(52.6)$ & \\
\hline Very good & $85(42.5)$ & $89(40.5)$ & $16(36.4)$ & $9(47.4)$ & \\
\hline \multicolumn{6}{|l|}{ Health status October 2020} \\
\hline Poor & $0(0)$ & $1(0.01)$ & $0(0)$ & $0(0)$ & 0.595 \\
\hline Fair & $18(9)$ & $16(7.3)$ & $5(11.4)$ & $0(0)$ & \\
\hline Good & $139(69.5)$ & $144(65.5)$ & $28(63.6)$ & $12(63.2)$ & \\
\hline Very good & $43(21.5)$ & $59(26.8)$ & $11(25.0)$ & $7(36.8)$ & \\
\hline
\end{tabular}

\footnotetext{
${ }^{1}$ Missing information 28 participants; ${ }^{2}$ missing information 6 participants; ${ }^{3}$ missing information 1 participant; ${ }^{4}$ missing information
} 21 participants.

A $32.9 \%$ of patients $(159 / 483)$ reported at least one complication (Table 4$)$ with a mean duration of $160.9 \pm 45.6$ days. The incidence of complications was higher in the B group $(50 \%)$, compared to the other $\mathrm{ABO}(31.2 \%)$ with a marginally non-significant difference $(p=0.072)$. In October $2020,81.8 \%$ of patients $(395 / 483)$ had recovered to their former health, but recovery was less frequent in the B group (70.5\%), compared to the other $\mathrm{ABO}$ $(82.9 \%)(p=0.047)$. In addition, $83.2 \%(402 / 483)$ reported that their health status was the same as before the illness; this was also lower in subjects of the B group (70.5\%) when compared to the subjects with other $\mathrm{ABO}(84.3 \%)(p=0.025)$.

In October 2020,53.4\% of patients reported at least one symptom $(258 / 483)$ without difference among $\mathrm{ABO}(p=0.586)$ (Table 4$)$. Reported symptoms of fatigue, abdominal pain, muscle pain, loss of smell/taste, headache, the difficulty to solve simple math operations, and skin lesions was higher in the $\mathrm{B}$ group. The $\mathrm{AB}$ group had a lower incidence of complications and reported symptoms than the $\mathrm{B}$ group, and compared with the $\mathrm{O}$ group, had high recovery and return health status as before. 
Table 4. Complications and now reported symptoms post-COVID-19 and ABO blood group. Borriana COVID-19 cohort 2020.

\begin{tabular}{|c|c|c|c|c|c|}
\hline \multirow[t]{2}{*}{ Variables } & \multicolumn{4}{|c|}{ ABO Blood Group } & \multirow[t]{2}{*}{$p$-Value } \\
\hline & $\begin{array}{c}\mathrm{O}(N=200) \\
N(\%)\end{array}$ & $\begin{array}{c}\mathrm{A}(N=220) \\
N(\%)\end{array}$ & $\begin{array}{c}\text { B }(N=44) \\
N(\%)\end{array}$ & $\begin{array}{c}\mathrm{AB}(N=19) \\
N(\%)\end{array}$ & \\
\hline Complications & $63(31.5)$ & $70(31.8)$ & $22(50.0)$ & $4(21.1)$ & 0.072 \\
\hline Recovery health ${ }^{1}$ & $166(83.0)$ & $179(81.4)$ & $31(70.5)$ & $19(100)$ & 0.047 \\
\hline Health the same as before ${ }^{2}$ & $173(86.5)$ & $178(80.9)$ & $32(72.7)$ & $19(100)$ & 0.025 \\
\hline Medical consultation ${ }^{3}$ & $19(9.6)$ & $23(10.6)$ & $5(11.9)$ & $0(0)$ & 0.539 \\
\hline Complications' duration (mean \pm standard deviation) & $163.0 \pm 47.0$ & $160.3 \pm 45.8$ & $158.3 \pm 44.6$ & $150.0 \pm 52.0$ & 0.919 \\
\hline \multicolumn{6}{|l|}{ Reported symptoms October 2020} \\
\hline Reported at least one symptom & $101(50.5)$ & $119(59.1)$ & $27(61.5)$ & $11(57.9)$ & 0.586 \\
\hline Fatigue & $32(16.0)$ & $40(18.1)$ & $13(29.6)$ & $2(10.5)$ & 0.187 \\
\hline Weakness & $14(7.0)$ & $17(7.7)$ & $6(13.6)$ & $3(15.8)$ & 0.240 \\
\hline Dyspnea & $13(6.5)$ & $16(7.3)$ & $3(6.8)$ & $1(5.3)$ & 0.986 \\
\hline Thorax oppression & $6(3.0)$ & $8(3.6)$ & $2(4.2)$ & $1(5.3)$ & 0.716 \\
\hline Cough & $13(6.5)$ & $10(4.6)$ & $2(4.6)$ & $1(5.3)$ & 0.810 \\
\hline Fever & $3(1.5)$ & $2(0.9)$ & $0(0)$ & $0(0)$ & 0.830 \\
\hline Throat pain & $9(4.5)$ & $15(6.8)$ & $4(9.1)$ & $0(0)$ & 0.424 \\
\hline Runny nose & $15(7.5)$ & $18(8.2)$ & $3(6.8)$ & $1(5.3)$ & 0.988 \\
\hline Loss smell/taste & $30(15)$ & 35 (15.9) & $11(35)$ & $2(10.5)$ & 0.390 \\
\hline Nausea/vomits & $4(2.0)$ & $2(0.9)$ & $1(2.3)$ & $0(0)$ & 0.561 \\
\hline Diarrhea & $8(4.0)$ & $11(5.0)$ & $1(2.3)$ & $1(5.3)$ & 0.817 \\
\hline Alimentary intolerance & $4(2.0)$ & $4(1.8)$ & $2(4.6)$ & $0(0)$ & 0.592 \\
\hline Abdominal pain & $9(4.5)$ & $6(7.3)$ & $4(9.1)$ & $1(5.3)$ & 0.179 \\
\hline Muscle pain & $21(10.5)$ & $27(12.3)$ & $8(18.2)$ & $0(0)$ & 0.196 \\
\hline Headache & $30(15)$ & $27(12.3)$ & $13(29.6)$ & $2(10.5)$ & 0.045 \\
\hline Hand/Foot pain & $16(8.0)$ & $18(8.2)$ & $2(4.6)$ & $0(0)$ & 0.664 \\
\hline Dizziness & $9(4.5)$ & $8(3.6)$ & $4(9.1)$ & $1(5.3)$ & 0.348 \\
\hline Ringing ears & $8(4.0)$ & $13(5.9)$ & $2(4.6)$ & $0(0)$ & 0.277 \\
\hline Disorder vision & $6(3.0)$ & $8(3.6)$ & $4(9.1)$ & $0(0)$ & 0.183 \\
\hline Insomnia & $25(12.5)$ & $22(10.0)$ & $7(15.9)$ & $2(10.5)$ & 0.634 \\
\hline Night sweats & $11(5.5)$ & $16(7.3)$ & $2(4.6)$ & $0(0)$ & 0.734 \\
\hline Depression & $9(4.5)$ & $5(2.3)$ & $1(2.3)$ & $0(0)$ & 0.593 \\
\hline Restlessness & $18(9.0)$ & $16(7.2)$ & $4(9.1)$ & $1(5.3)$ & 0.913 \\
\hline Difficulty concentration & $10(5.0)$ & $7(3.2)$ & $3(6.8)$ & $0(0)$ & 0.544 \\
\hline Anxiety & $18(9.0)$ & $21(9.6)$ & $4(9.1)$ & $1(5.3)$ & 0.991 \\
\hline Mental confusion & $9(4.5)$ & $6(2.7)$ & $1(2.3)$ & $0(0)$ & 0.764 \\
\hline Difficulty articulating words & $1(0.5)$ & $3(1.4)$ & $2(4.6)$ & $0(0)$ & 0.181 \\
\hline Difficulty to solve simple math operations & $2(1.0)$ & 0 & $2(4.6)$ & $0(0)$ & 0.030 \\
\hline Skin lesions & $11(5.0)$ & $8(3.6)$ & $6(13.6)$ & $0(0)$ & 0.059 \\
\hline Hair lost & $48(24.0)$ & $51(23.2)$ & $15(34.1)$ & $5(26.3)$ & 0.472 \\
\hline
\end{tabular}

\footnotetext{
${ }^{1}$ Missing information 1 participant; ${ }^{2}$ missing information 1 participant; ${ }^{3}$ missing information 5 participants.
}

We show adjusted incidence rate ( $\mathrm{aIR}$ ) and adjusted RR (aRR) of complications and reported symptoms in Table 5 . The $\mathrm{B}$ group presented higher aIR than the other $\mathrm{ABO}$ in complications, lower recovery, and return health status as before with aRR of 1.68 (95\% CI $1.24-2.27), 0.86$ (0.70-1.03), and 0.85 (0.71-1.04). 
Table 5. Adjusted incidence rate (aIR), adjusted relative risk (aRR), and 95\% confidence interval (CI) by inverse probability weight regression of complications, and reported symptoms of ABO blood groups. Borriana COVID-19 cohort 2020.

\begin{tabular}{|c|c|c|c|c|}
\hline Variables & $\operatorname{aIR}^{1}(\%)$ & aRR $^{1}$ & $95 \%$ CI & $p$-Value \\
\hline \multicolumn{5}{|c|}{ Complications } \\
\hline $\mathrm{O}$ & 32.3 & 1.00 & & \\
\hline A & 32.6 & 1.01 & $0.77-1.33$ & 0.950 \\
\hline $\mathrm{B}$ & 54.1 & 1.68 & $1.24-2.27$ & 0.001 \\
\hline $\mathrm{AB}$ & 27.6 & 0.24 & $0.01-4.90$ & 0.351 \\
\hline \multicolumn{5}{|c|}{ Recovery of illness } \\
\hline $\mathrm{O}$ & 83.1 & 1.00 & & \\
\hline A & 81.4 & 0.98 & $0.89-1.07$ & 0.621 \\
\hline $\mathrm{B}$ & 72.8 & 0.86 & $0.71-1.04$ & 0.127 \\
\hline $\mathrm{AB}$ & 100.0 & 1.20 & $1.13-1.28$ & 0.000 \\
\hline \multicolumn{5}{|c|}{ Health the same as before } \\
\hline $\mathrm{O}$ & 86.4 & 1.00 & & \\
\hline A & 79.9 & 0.93 & $0.85-1.01$ & 0.077 \\
\hline $\mathrm{B}$ & 73.7 & 0.85 & $0.70-1.03$ & 0.103 \\
\hline $\mathrm{AB}$ & 100.0 & 1.16 & $1.10-1.22$ & 0.000 \\
\hline \multicolumn{5}{|c|}{ Reported symptoms } \\
\hline \multicolumn{5}{|c|}{ Fatigue } \\
\hline $\mathrm{O}$ & 16.2 & 1.00 & & \\
\hline $\mathrm{A}$ & 19.9 & 1.22 & $0.81-1.85$ & 0.331 \\
\hline $\mathrm{B}$ & 29.0 & 1.79 & $1.08-2.95$ & 0.023 \\
\hline $\mathrm{AB}$ & 2.6 & 0.16 & $0.01-5.23$ & 0.305 \\
\hline \multicolumn{5}{|l|}{ Muscle pain } \\
\hline $\mathrm{O}^{\mathrm{r}}$ & 9.8 & 1.00 & & \\
\hline A & 13.0 & 1.32 & $0.79-2.22$ & 0.292 \\
\hline B & 21.1 & 2.06 & $1.10-3.84$ & 0.023 \\
\hline $\mathrm{AB}$ & 0.0 & $\mathrm{NC}^{2}$ & & $\mathrm{NC}^{2}$ \\
\hline \multicolumn{5}{|c|}{ Loss smell/taste } \\
\hline $\mathrm{O}$ & 14.8 & 1.00 & & \\
\hline A & 16.5 & 1.11 & $0.71-1.74$ & 0.652 \\
\hline $\mathrm{B}$ & 26.0 & 1.75 & $0.95-3.23$ & 0.070 \\
\hline $\mathrm{AB}$ & 15.1 & & $0.01-9.28$ & 0.524 \\
\hline \multicolumn{5}{|l|}{ Headache } \\
\hline $\mathrm{O}$ & 13.9 & 1.00 & & \\
\hline A & 12.9 & 0.93 & $0.57-1.50$ & 0.751 \\
\hline B & 36.4 & 2.61 & $1.58-4.31$ & 0.000 \\
\hline $\mathrm{AB}$ & 20.7 & 1.49 & $0.60-3.71$ & 0.392 \\
\hline \multicolumn{5}{|c|}{ Skin's lesions } \\
\hline $\mathrm{O}$ & 5.5 & 1.00 & & \\
\hline A & 3.4 & 0.71 & $0.29-1.72$ & 0.448 \\
\hline B & 14.1 & 2.46 & $0.98-6.20$ & 0.056 \\
\hline $\mathrm{AB}$ & 0 & $\mathrm{NC}^{2}$ & & $\mathrm{NC}^{2}$ \\
\hline \multicolumn{5}{|c|}{ Disorder vision } \\
\hline $\mathrm{O}$ & 3.0 & 1.00 & & \\
\hline $\mathrm{A}$ & 3.8 & 1.29 & $0.46-3.61$ & 0.624 \\
\hline $\mathrm{B}$ & 12.8 & 4.26 & $1.33-13.60$ & 0.014 \\
\hline $\mathrm{AB}$ & 0 & $\mathrm{NC}^{2}$ & & $\mathrm{NC}^{2}$ \\
\hline \multicolumn{5}{|l|}{ Brain fog 3} \\
\hline $\mathrm{O}$ & 12.3 & 1.00 & & \\
\hline A & 8.4 & 0.69 & $0.38-1.20$ & 0.188 \\
\hline $\mathrm{B}$ & 18.1 & 1.46 & $0.75-2.73$ & 0.266 \\
\hline $\mathrm{AB}$ & 5.9 & 0.48 & $0.13-1.71$ & 0.257 \\
\hline \multicolumn{5}{|c|}{ Medical consultation acute illness } \\
\hline $\mathrm{O}$ & 42.5 & 1.00 & & \\
\hline A & 44.5 & 1.05 & $0.85-1.29$ & 0.678 \\
\hline $\mathrm{B}$ & 56.7 & 1.33 & $1.01-1.75$ & 0.041 \\
\hline $\mathrm{AB}$ & 31.9 & 0.75 & $0.44-1.28$ & 0.294 \\
\hline
\end{tabular}

${ }_{1}$ Adjusted for age, sex, smoking, alcohol consumption, body mass index, physical exercise, person cough mass gathering events (MGE), contact COVID-19 case, family COVID-19 case, and attendance MGE $\geq 2$ and over ${ }^{2} \mathrm{NC}=$ not computable. ${ }^{3}$ Brain fog $=$ difficulty concentration + mental confusion + restlessness + difficulty articulating words + difficulty simple math operations.

Compared with the O-group subjects, the B-group subjects reported more frequently symptoms of fatigue $(29.0 \%$ versus $16.2 \%$ aRR $=1.7995 \%$ CI $1.08-2.95)$, muscle pain $(21.1 \%$ versus $9.8 \%$ aRR $=2.0695 \% \mathrm{CI} 1.10-3.84)$, headache $(36.4 \%$ versus $13.9 \%$ aRR $=2.6195 \% \mathrm{CI}$ 
$1.58-4.31)$, disorder vision (12.8\% versus $3.0 \%$ aRR $=4.26,95 \%$ CI 1.33-13.60), and medical consultation in the acute illness (56.7\% versus $42.5 \%$ aRR $=1.3395 \%$ CI $1.01-1.75)$. Loss of the smell/taste was higher in the B group but with a marginally non-significant difference (26.0\% versus $14.8 \%$ aRR $=1.75,95 \%$ CI $0.95-3.23)$. Brain fog, as a summary of mental symptoms (Table 5), was higher in the B group but not significant (18.1\% versus $12.3 \%$ aRR $=1.4695 \%$ CI 0.75-2.73). On the other hand, the AB group's small size prevented obtaining valid estimations.

\section{Discussion}

Our results suggest that $\mathrm{ABO}$ had not an appreciable impact on the incidence of COVID19 infection, with the B-group subjects experiencing a higher incidence of complications and reported symptoms than the $\mathrm{O}$ group at six months after the acute COVID-19 illness.

Regarding the incidence of SARS-CoV-2 infection in $\mathrm{ABO}$, our results are in line with several studies where no effect of $\mathrm{ABO}$ could be found with suitable controls for comparison $[17,18,34,35]$. The protection of the $\mathrm{O}$ group among $\mathrm{ABO}$, which could be found in several studies $[29,36,37]$, is overcome following the Ellis model of ABO incompatibility and SARS-CoV-2 transmission [38]; in the first stages of the epidemic, the non-O groups are more infected, but after, when the majority of the population is infected, the $\mathrm{O}$ group has a similar infection rate.

Most ABO studies have been focused on the incidence and severity of COVID-19 [17], and few studies on symptoms and complications post-COVID-19. Some studies found that the $\mathrm{O}$ group offered protection against COVID-19 infection, whereas A-group subjects experienced a higher risk of adverse outcomes $[12,13,39,40]$, with more cardiovascular complications in the A-group subjects [41], whereas subjects in the B group experienced more severe illnesses and higher fatality rates $[11,15,22,41,42]$, but no ABO blood group differences in mortality were found in other studies [43-45].

In our study, the B-group subjects reported more symptoms and severity of the disease than the other groups. This pattern has been associated with post-COVID-19 complications $[46,47]$. Complications and reported symptoms here are concordant with the post-COVID-19 disease despite a mild illness in most patients. Hair loss, fatigue, smell/taste loss, headache, muscle pain, insomnia, and anxiety symptoms agree with the post-COVID-19 disease definition [48,49]. In general, physical symptoms (fatigue, muscle pain, headache, lost smell/taste, and vision disorder) had a higher incidence in the B group than mental symptoms (anxiety, depression, insomnia) when compared with other ABO. No difference in complications and reported symptoms were found between $\mathrm{O}$ and $\mathrm{A}$ groups, in contrast with other studies [23,50-52].

Le Pendu et al. [53], in a review of the association between ABO and COVID-19, concluded that the $\mathrm{O}$ group provided some protection in comparison with non-O groups, and this could be mediated either by natural anti-A and anti-B antibodies or by a lower efficiency of furin cleavage in the O group [54,55]. In addition, Bloch et al. [56] found that blood donors of group B had higher neutralizing antibody titers than the other ABO. According to this study [56], a possibility is cross reactivity of the virus and the B antigen, and this increases antibody production, but the cause is unknown. In our previous study [26], the $\mathrm{O}$ group presented lower persistence of anti-SARS-CoV-2 antibodies than the non-O groups, but we found no difference in antibody levels between $\mathrm{ABO}$ blood groups.

Concerning infectious diseases, the $\mathrm{O}$ group could have special protection derived from anti-A/anti-B actions of humoral innate immunity $[57,58]$. In addition, the $\mathrm{O}$ group had some physiological advantages such as endurance running, compared with non-Ogroups [59]. ABO differences in respect to health and disease are not entirely understood. Still, some studies found that $\mathrm{O}$ group subjects have lower thrombosis and more hypertension disease than no-O groups, and the A group has more risk of cardiovascular disease [60-62].

The study had some important aspects, including a population-based prospective cohort design, high participation rate, a follow-up of 6 months, and control of potential 
confounding factors, considering that the exposure to the virus and the duration time could play a crucial role in the clinical course of the disease [63].

The study has some limitations; measured manifestations were reported by questionnaire, so we could not discard information, recall bias, and residual confounding. COVID-19 is a new disease, and some aspects could not be considered in advance. Most patients had a mild illness, and they may not be representative of the COVID-19 patients. A small sample of the $\mathrm{AB}$ group prevented definitive estimations in this blood group. The study was focused on COVID-19 patients, and we could not compare our findings with the COVID-19 negative member of the cohort.

Our results support the active medical follow-up of COVID-19 patients considering the high level of symptoms persistence [64]. In our study, the B group appears associated with a higher risk of prolonged symptoms, whereas the $\mathrm{O}$ group subjects experienced lower affectation. Considering that there are few studies on ABO blood groups and COVID19 complications, further research is justified to improve our understanding of the ABO relationship with COVID-19 [65-67].

\section{Conclusions}

ABO blood group patients presented significant differences in post-COVID-19 complications with a more severe course observed in the B group. Additional research is justified to confirm our results.

Author Contributions: Conceptualization, S.D.-M., A.A.-P., M.R.P.-S., L.G.-L., D.J.-S., L.A.-E., U.C.A., S.F.-R. and M.S.-U.; methodology, A.A.-P., S.D.-M., J.P.-B., P.V.-U., M.L.-P., A.D.R.-G., S.F.-R., M.S.-U., G.F.-A., L.A.-E., G.B.-M., U.C.-A., C.D.-B. and M.M.-B.; software A.A.-P., M.R.P.-S., J.P.-B. and M.C.L.-D.; validation, J.P.-B., C.N.-R. and M.R.P.-S.; formal analysis, M.R.P.-S., A.A.-P., J.P.-B. and S.D.-M.; investigation, S.D.-M., M.R.P.-S., P.V.-U., M.L.-P., A.D.R.-G., S.F.-R., G.F.-A., M.S.-U., L.A.-E., G.B.-M., B.C.-F., U.C.-A., C.D.-B., M.F.-C., L.G.-L., D.J.-S., M.C.L.-D., M.D.L.-V., M.M.-B., C.N.-R., R.R.-P. and S.V.-L.; resources, S.D.-M., G.F.-A., G.B.-M., B.C.-F., M.F.-C., L.G.-L., M.C.L.D., M.D.L.-V., M.L.-P., C.N.-R., R.R.-P. and S.V.-L.; data curation, A.A.-P., J.P.-B., P.V.-U., M.L.-P., A.D.R.-G. and M.R.P.-S.; writing - original draft preparation, A.A.-P., J.P.-B., M.R.P.-S., M.C.L.-D., S.D.-M. and S.F.-R.; writing-review and editing, J.P.-B., A.A.-P., S.D.-M., U.C.-A., M.F.-C. and M.C.L.D.; visualization, J.P.-B., D.J.-S., L.A.-E. and L.G.-L.; supervision M.R.P.-S. and M.C.L.-D.; project administration, S.D.-M., A.A.-P., M.C.L.-D. and G.F.-A.; funding acquisition, S.D.-M., M.C.L.-D. and A.A.-P. All authors have read and agreed to the published version of the manuscript.

Funding: This research received no external funding.

Institutional Review Board Statement: The study was conducted according to the guidelines of the Declaration of Helsinki. The study was part of the public health surveillance as a prolongation of the COVID-19 outbreak in the Falles festival in Borriana control measures, which was exempted from Ethics Review Board approval's protocol according to the Spanish legislation, including (1) the General Law of Health, (2) the Law of Cohesion and Quality of the National System of Health, and (3) the Law General of Public Health. The study was approved by the director of the Public Health Center of Castellon and the management of the Health Department of La Plana. In addition, (4) the cohort was following to respond to a new disease, the COVID-19 pandemic. 1. Ley 14/1986, de 25 de abril, General de Sanidad (Law General of Health). Available from https:/ /www.boe.es/eli/es/ 1/1986/04/25/14/con, Accesed date 2 August 2021. 2. Ley 16/2003, de 28 de mayo, de cohesión y calidad del Sistema Nacional de Salud. (Law of Cohesion and Quality of the National System of Health). Available from https://www.boe.es/eli/es/1/2003/05/28/16, Accesed date 2 August 2021. 3. Ley 33/2011, de 4 de octubre, General de Salud Pública (Law General of Public Health). Available from https:/ / www.boe.es/eli/es/1/2011/10/04/33/con, Accesed date 2 August 2021. 4. Ministerio de Sanidad Acordado en Consejo Interterritorial del Sistema Nacional de Salud el 16 de julio de 2020. Plan de respuesta temprana en un escenario de control de la pandemia por el COVID-19.pdf "Ministry of Health Agreed in the Interterritorial Council of the National Health System on 16 July 2020". Early response plan in a COVID-19 pandemic control scenario.pdf.

Informed Consent Statement: Informed consent was obtained from all subjects involved in the study. 
Data Availability Statement: Data of this study can be consulted if the authors are requested. Dataset: borrianacohort.dta.

Acknowledgments: We thank the participants of the cohort and the Borriana's Falles organization for the support that made it possible to perform this study. In addition, we appreciate the assistance and support of Roser Blasco-Gari, Helena Buj-Jorda, Israel Borras-Acosta, Lucia Castell-Agusti, Mercedes De Francia-Valero, Maria Domènech-Molinos, Marc Garcia, Maria Gil-Fortuño, Elena GrañanaToran, Noelia Hernández-Perez, Laura Lopez-Diago, Salvador Martinez-Parra, Sara Moner-Marin, Silvia Pesudo-Calatayud, Lara Sabater-Hernández, Maria Luisa Salve-Martinez, Irene Suarez-Linares, Juan José Ventura-Buchardo, and Alberto Yagüe-Muñoz to carry out the study.

Conflicts of Interest: The authors declare no conflict of interest.

\section{References}

1. Aiyegbusi, O.L.; Hughes, S.E.; Turner, G.; Rivera, S.C.; McMullan, C.; Chandan, J.S.; Haroon, S.; Price, G.; Davies, E.H.; Nirantharakumar, K.; et al. Symptoms, complications and management of long COVID: A review. J. R. Soc. Med. 2021, 15, 1410768211032850.

2. Davis, H.E.; Assafa, G.S.; McCorkell, L.; Wei, H.; Low, R.J.; Re'em, Y.; Redfield, S.; Jared PAustin, J.P.; Akrami, A. Characterizing long COVID in an international cohort: 7 months of symptoms and their impact. EClinicalMedicine 2021, 38, 101019. [CrossRef]

3. Osmanov, I.M.; Spiridonova, E.; Bobkova, P.; Gamirova, A.; Shikhaleva, A.; Andreeva, M.; Blyuss, O.; El-Taravi, Y.; DunnGalvin, A.; Comberiati, P.; et al. Risk factors for long COVID in previously hospitalised children using the ISARIC Global follow-up protocol: A prospective cohort study. Eur. Respir. J. 2021, 2101341. [CrossRef]

4. Salzberger, B.; Buder, F.; Lampl, B.; Ehrenstein, B.; Hitzenbichler, F.; Holzmann, T.; Schmidt, B.; Hanses, F. Epidemiology of SARS-CoV-2. Infection 2021, 49, 233-239. [CrossRef]

5. Carethers, J.M. Insights into disparities observed with COVID-19. J. Intern. Med. 2021, 289, 463-473. [CrossRef]

6. Li, J.; Wang, X.; Chen, J.; Cai, Y.; Deng, A.; Yang, M. Association between ABO blood groups and risk of SARS-CoV-2 pneumonia. Br. J. Haematol. 2020, 190, 24-27. [CrossRef] [PubMed]

7. Zhao, J.; Yang, Y.; Huang, H.; Li, D.; Gu, D.; Lu, X.; Zhang, Z.; Liu, L.; Liu, T.; Liu, Y.; et al. Relationship between the ABO Blood Group and the COVID-19 Susceptibility. Clin. Infect. Dis. 2020, 73, 328-331. [CrossRef] [PubMed]

8. Wu, B.B.; Gu, D.Z.; Yu, J.N.; Yang, J.; Shen, W.Q. Association between ABO blood groups and COVID-19 infection, severity and demise: A systematic review and meta-analysis. Infect. Genet. Evol. 2020, 84, 104485. [CrossRef] [PubMed]

9. Zhang, Y.; Garner, R.; Salehi, S.; La Rocca, M.; Duncan, D. Association between ABO blood types and coronavirus disease 2019 (COVID-19), genetic associations, and underlying molecular mechanisms: A literature review of 23 studies. Ann. Hematol. 2021, 100, 1123-1132. [CrossRef]

10. AbdelMassih, A.F.; Mahrous, R.; Taha, A.; Saud, A.; Osman, A.; Kamel, B.; Yacoub, E.; Menshawey, E.; Ismail, H.A.; Aita, L.; et al. The potential use of ABO blood group system for risk stratification of COVID-19. Med. Hypotheses 2020, 145, 110343. [CrossRef] [PubMed]

11. Liu, N.; Zhang, T.; Ma, L.; Zhang, H.; Wang, H.; Wei, W.; Pei, H.; Li, H. The impact of ABO blood group on COVID-19 infection risk and mortality: A systematic review and meta-analysis. Blood Rev. 2021, 48, 100785. [CrossRef]

12. Ellinghaus, D.; Degenhardt, F.; Bujanda, L.; Buti, M.; Albillos, A.; Invernizzi, P.; Fernández, J.; Prati, D.; Baselli, G.; Asselta, R.; et al. Genomewide Association Study of Severe COVID-19 with Respiratory Failure. N. Engl. J. Med. 2020, 383, 1522-1534. [PubMed]

13. Muñiz-Diaz, E.; Llopis, J.; Parra, R.; Roig, I.; Ferrer, G.; Grifols, J.; Millán, A.; Ene, G.; Ramiro, L.; Maglio, L.; et al. Relationship between the ABO blood group and COVID-19 susceptibility, severity and mortality in two cohorts of patients. Blood Transfus. 2021, 19, 54-63. [PubMed]

14. Rahim, F.; Amin, S.; Bahadur, S.; Noor, M.; Mahmood, A.; Gul, H. ABO/Rh-D Blood types and susceptibility to Corona Virus Disease-19 in Peshawar, Pakistan. Pak. J. Med. Sci. 2021, 37, 4-8. [PubMed]

15. Padhi, S.; Suvankar, S.; Dash, D.; Panda, V.K.; Pati, A.; Panigrahi, J.; Panda, A.K. ABO blood group system is associated with COVID-19 mortality: An epidemiological investigation in the Indian population. Transfus. Clin. Biol. 2020, 27, 253-258. [CrossRef]

16. Göker, H.; Aladağ Karakulak, E.; Demiroğlu, H.; Ayaz Ceylan, Ç.M.; Büyükaşik, Y.; Inkaya, A.Ç.; Aksu, S.; Sayinalp, N.; Haznedaroğlu, I.C.; Uzun, Ö.; et al. The effects of blood group types on the risk of COVID-19 infection and its clinical outcome. Turk. J. Med. Sci. 2020, 50, 679-683. [CrossRef]

17. Anderson, J.L.; May, H.T.; Knight, S.; Bair, T.L.; Muhlestein, J.B.; Knowlton, K.U.; Horne, B.D. Association of sociodemographic factors and blood group type with risk of COVID-19 in a US population. JAMA Netw. Open 2021, 4, e217429. [CrossRef]

18. Boudin, L.; Janvier, F.; Bylicki, O.; Dutasta, F. ABO blood groups are not associated with risk of acquiring the SARS-CoV-2 infection in young adults. Haematologica 2020, 105, 2841-2843. [CrossRef] [PubMed]

19. Bhandari, P.; Durrance, R.J.; Bhuti, P.; Salama, C. Analysis of ABO and Rh blood type association with acute COVID-19 infection in hospitalized patients: A superficial association among a multitude of established confounders. J. Clin. Med. Res. 2020, 12, 809-815. [CrossRef]

20. Niles, J.K.; Karnes, H.E.; Dlott, J.S.; Kaufman, H.W. Association of ABO/Rh with SARS-CoV-2 positivity: The role of race and ethnicity in a female cohort. Am. J. Hematol. 2021, 96, E23-E26. [CrossRef] 
21. Sardu, C.; Marfella, R.; Maggi, P.; Messina, V.; Cirillo, P.; Codella, V.; Gambardella, J.; Sardu, A.; Gatta, G.; Santulli, G.; et al. Implications of AB0 blood group in hypertensive patients with COVID-19. BMC Cardiovasc. Disord. 2020, 20, 373. [CrossRef] [PubMed]

22. Ray, J.G.; Schull, M.J.; Vermeulen, M.J.; Park, A.L. Association Between ABO and Rh blood groups and SARS-CoV-2 infection or severe COVID-19 illness: A population-based cohort study. Ann. Intern. Med. 2021, 174, 308-315. [CrossRef] [PubMed]

23. Hoiland, R.L.; Fergusson, N.A.; Mitra, A.R.; Griesdale, D.E.G.; Devine, D.V.; Stukas, S.; Cooper, J.; Thiara, S.; Foster, D.; Chen, L.Y.C.; et al. The association of ABO blood group with indices of disease severity and multiorgan dysfunction in COVID-19. Blood Adv. 2020, 4, 4981-4989. [CrossRef] [PubMed]

24. Dal, M.S.; Ata, N.; Altuntaş, F.; Başci, S.; Yiğenoğlu, T.N.; Korkmaz, S.; Namdaroğlu, S.; Baştürk, A.; Hacibekiroğlu, T.; Doğu, M.H.; et al. COVID-19 clinical course and blood groups: Turkish population-based study. Turk. J. Med. Sci. 2021, 51, 1659-1664. [CrossRef] [PubMed]

25. Domènech-Montoliu, S.; Pac-Sa, M.R.; Vidal-Utrillas, P.; Latorre-Poveda, M.; Del Rio-González, A.; Ferrando-Rubert, S.; FerrerAbad, G.; Sánchez-Urbano, M.; Aparisi-Esteve, L.; Badenes-Marques, G.; et al. Mass gathering events and COVID-19 transmission in Borriana (Spain): A retrospective cohort study. PLoS ONE 2021, 16, e0256747. [CrossRef] [PubMed]

26. Domènech-Montoliu, S.; Puig-Barberà, J.; Pac-Sa, M.R.; Vidal-Utrillas, P.; Latorre-Poveda, M.; Del Rio-González, A.; FerrandoRubert, S.; Ferrer-Abad, G.; Sánchez-Urbano, M.; Aparisi-Esteve, L.; et al. Persistence of anti-SARS-CoV-2 antibodies six months after infection in an outbreak with five hundred COVID-19 cases in Borriana (Spain): A prospective cohort study. COVID 2021, 1, 71-82. [CrossRef]

27. Egger, M.; Bundschuh, C.; Wiesinger, K.; Gabriel, C.; Clodi, M.; Mueller, T.; Dieplinger, B. Comparison of the Elecsys ${ }^{\circledR A n t i-}$ SARSCoV-2 immunoassay with the EDI ${ }^{\mathrm{TM}}$ enzyme linked immunosorbent assays for the detection of SARS-CoV-2 antibodies in human plasma. Clin. Chim. Acta 2020, 509, 18-21. [CrossRef]

28. Lapierre, Y.; Rigal, D.; Adam, J.; Josef, D.; Meyer, F.; Greber, S.; Drot, C. The gel test: A new way to detect red cell antigen-antibody reactions. Transfusion 1990, 30, 109-113. [CrossRef]

29. Zalba Marcos, S.; Antelo, M.L.; Galbete, A.; Etayo, M.; Ongay, E.; García-Erce, J.A. Infection and thrombosis associated with COVID-19: Possible role of the ABO blood group. Med. Clin. (Engl. Ed.) 2020, 155, 340-343. [CrossRef]

30. Nogareda-Barbudo, A. Grupos sanguineos en la población activa española. An. Med. Cir. 1964, 45, 115-123.

31. Greenland, S.; Pearl, J.; Robins, J.M. Causal diagrams for epidemiologic research. Epidemiology 1999, 10, 37-48. [CrossRef] [PubMed]

32. Textor, J.; van der Zander, B.; Gilthorpe, M.S.; Liskiewicz, M.; Ellison, G.T. Robust causal inference using directed acyclic graphs: The R package 'dagitty'. Int. J. Epidemiol. 2016, 45, 1887-1894. [CrossRef]

33. Robins, J.M.; Hernán, M.A.; Brumback, B. Marginal structural models and causal inference in epidemiology. Epidemiology 2000, 11, 550-560. [CrossRef]

34. Covali, R.; Socolov, D.; Pavaleanu, I.; Carauleanu, A.; Boiculese, V.L.; Socolov, R. SARS-CoV-2 Infection susceptibility of pregnant patients at term regarding ABO and Rh blood groups: A cohort study. Medicina (Kaunas) 2021, 57, 499. [CrossRef] [PubMed]

35. Dzik, S.; Eliason, K.; Morris, E.B.; Kaufman, R.M.; North, C.M. COVID-19 and ABO blood groups. Transfusion 2020, 60, 1883-1884. [CrossRef] [PubMed]

36. Valenti, L.; Villa, S.; Baselli, G.; Temporiti, R.; Bandera, A.; Scudeller, L.; Prati, D. Association of ABO blood group and secretor phenotype with severe COVID-19. Transfusion 2020, 60, 3067-3070. [CrossRef] [PubMed]

37. Deleers, M.; Breiman, A.; Daubie, V.; Maggetto, C.; Barreau, I.; Besse, T.; Clémenceau, B.; Ruvoën-Clouet, N.; Fils, J.F.; Maillart, E.; et al. COVID-19 and blood groups: ABO antibody levels may also matter. Int. J. Infect. Dis. 2021, 104, 242-249. [CrossRef]

38. Ellis, P.J.I. Modelling suggests ABO histo-incompatibility may substantially reduce SARS-CoV-2 transmission. Epidemics 2021, 35, 100446. [CrossRef]

39. Fan, Q.; Zhang, W.; Li, B.; Li, D.J.; Zhang, J.; Zhao, F. Association between ABO blood group system and COVID-19 susceptibility in Wuhan. Front. Cell. Infect. Microbiol. 2020, 10, 404. [CrossRef]

40. Bari, A.; Ch, A.; Hareem, S.; Bano, I.; Rashid, J.; Sadiq, M. Association of blood groups with the severity and outcome of COVID-19 infection in children. J. Coll Physicians Surg. Pak. 2021, 30, S57-S59.

41. Mankelow, T.J.; Singleton, B.K.; Moura, P.L.; Stevens-Hernandez, C.J.; Cogan, N.M.; Gyorffy, G.; Kupzig, S.; Nichols, L.; Asby, C.; Pooley, J.; et al. Blood group type A secretors are associated with a higher risk of COVID-19 cardiovascular disease complications. EJHaem 2021, 2, 175-187. [CrossRef] [PubMed]

42. Zietz, M.; Zucker, J.; Tatonetti, N.P. Associations between blood type and COVID-19 infection, intubation, and death. Nat. Commun. 2020, 11, 5761. [CrossRef] [PubMed]

43. Kumar, G.; Nanchal, R.; Hererra, M.; Sakhuja, A.; Patel, D.; Meersman, M.; Dalton, D.; Guddati, A.K. Does ABO blood groups affect outcomes in hospitalized COVID-19 patients? J. Hematol. 2021, 10, 98-105. [CrossRef] [PubMed]

44. Lehrer, S.; Rheinstein, P.H. ABO blood groups, COVID-19 infection and mortality. Blood Cells Mol. Dis. 2021, 89, 102571. [CrossRef]

45. Mullins, J.; Al-Tarbsheh, A.H.; Chieng, H.; Chaukiyal, P.; Ghalib, S.; Jain, E.; Dawani, O.; Santelises Robledo, F.M.; Chong, W.H.; Feustel, P.J.; et al. The association of ABO blood type with the risk and severity of COVID-19 infection. Am. J. Blood Res. 2021, 16, 53-58. 
46. Mahmud, R.; Rahman, M.M.; Rassel, M.A.; Monayem, F.B.; Sayeed, S.K.J.B.; Islam, M.S.; Islam, M.M. Post-COVID-19 syndrome among symptomatic COVID-19 patients: A prospective cohort study in a tertiary care center of Bangladesh. PLoS ONE 2021, 16, e0249644. [CrossRef]

47. Sudre, C.H.; Murray, B.; Varsavsky, T.; Graham, M.S.; Penfold, R.S.; Bowyer, R.C.; Pujol, J.C.; Klaser, K.; Antonelli, M.; Canas, L.S.; et al. Attributes and predictors of long COVID. Nat. Med. 2021, 27, 626-631. [CrossRef]

48. Shanbehzadeh, S.; Tavahomi, M.; Zanjari, N.; Ebrahimi-Takamjani, I.; Amiri-Arimi, S. Physical and mental health complications post-COVID-19: Scoping review. J. Psychosom. Res. 2021, 147, 110525. [CrossRef]

49. Xiong, Q.; Xu, M.; Li, J.; Liu, Y.; Zhang, J.; Xu, Y.; Dong, W. Clinical sequelae of COVID-19 survivors in Wuhan, China: A single-centre longitudinal study. Clin. Microbiol. Infect. 2021, 27, 89-95. [CrossRef]

50. Wu, Y.; Feng, Z.; Li, P.; Yu, Q. Relationship between ABO blood group distribution and clinical characteristics in patients with COVID-19. Clin. Chim. Acta 2020, 509, 220-223. [CrossRef]

51. Nauffal, V.; Achanta, A.; Goldhaber, S.Z.; Piazza, G. Association of ABO blood group type with cardiovascular events in COVID-19. J Thromb. Thrombolysis 2021, 51, 584-586. [CrossRef] [PubMed]

52. Golinelli, D.; Boetto, E.; Maietti, E.; Fantini, M.P. The association between ABO blood group and SARS-CoV-2 infection: A meta-analysis. PLOS ONE 2020, 15, e0239508. [CrossRef]

53. Le Pendu, J.; Breiman, A.; Rocher, J.; Dion, M.; Ruvoën-Clouet, N. ABO blood types and COVID-19: Spurious, Anecdotal, or truly important relationships? A reasoned review of available data. Viruses 2021, 13, 160. [CrossRef] [PubMed]

54. Gérard, C.; Maggipinto, G.; Minon, J.M. COVID-19 and ABO blood group: Another viewpoint. Br. J. Haematol. 2020, 190, e93-e94. [CrossRef] [PubMed]

55. Breiman, A.; Ruvën-Clouet, N.; Le Pendu, J. Harnessing the natural anti-glycan immune response to limit the transmission of enveloped viruses such as SARS-CoV-2. PLoS Pathog. 2020, 16, e1008556. [CrossRef] [PubMed]

56. Bloch, E.M.; Patel, E.U.; Marshall, C.; Littlefield, K.; Goel, R.; Grossman, B.J.; Winters, J.L.; Shrestha, R.; Burgess, I.; Laeyendecker, O.; et al. ABO blood group and SARS-CoV-2 antibody response in a convalescent donor population. Vox Sang. 2021, 116, 766-773. [CrossRef]

57. Arend, P. Position of human blood group $\mathrm{O}(\mathrm{H})$ and phenotype-determining enzymes in growth and infectious disease. Ann. N. Y. Acad. Sci. 2018, 1425, 5-18. [CrossRef]

58. Arend, P. Why blood group A individuals are at risk whereas blood group O individuals are protected from SARS-CoV-2 (COVID-19) infection: A hypothesis regarding how the virus invades the human body via $\mathrm{ABO}(\mathrm{H})$ blood group-determining carbohydrates. Immunobiology 2021, 226, 152027. [CrossRef]

59. Lippi, G.; Gandini, G.; Salvagno, G.L.; Skafidas, S.; Festa, L.; Danese, E.; Montagnana, M.; Sanchis-Gomar, F.; Tarperi, C.; Schena, F. Influence of ABO blood group on sports performance. Ann. Transl. Med. 2017, 5, 255. [CrossRef]

60. Groot, H.E.; Villegas Sierra, L.E.; Said, M.A.; Lipsic, E.; Karper, J.C.; van der Harst, P. Genetically determined ABO blood group and its associations with health and disease. Arter. Thromb. Vasc. Biol. 2020, 40, 830-838. [CrossRef]

61. Dai, X. ABO blood group predisposes to COVID-19 severity and cardiovascular diseases. Eur J. Prev Cardiol. 2020, $27,1436-1437$. [CrossRef] [PubMed]

62. Ahmed, I.; Quinn, L.; Tan, B.K. COVID-19 and the ABO blood group in pregnancy: A tale of two multiethnic cities. Int. J. Lab. Hematol. 2021, 43, e45-e47. [CrossRef] [PubMed]

63. Calisti, R. SARS-CoV-2: Exposure to high external doses as determinants of higher viral loads and of increased risk for COVID-19. A systematic review of the literature. Epidemiol. Prev. 2020, 44, 152-159. [PubMed]

64. De Lorenzo, R.; Conte, C.; Lanzani, C.; Benedetti, F.; Roveri, L.; Mazza, M.G.; Brioni, E.; Giacalone, G.; Canti, V.; Sofia, V.; et al. Residual clinical damage after COVID-19: A retrospective and prospective observational cohort study. PLoS ONE 2020, 15, e0239570. [CrossRef] [PubMed]

65. Yamamoto, F.; Yamamoto, M.; Muñiz-Diaz, E. Blood group ABO polymorphism inhibits SARS-CoV-2 infection and affects COVID-19 progression. Vox Sang. 2021, 116, 15-17. [CrossRef]

66. Goel, R.; Bloch, E.M.; Pirenne, F.; Al-Riyami, A.Z.; Crowe, E.; Dau, L.; Land, K.; Townsend, M.; Jecko, T.; Rahimi-Levene, N.; et al. ABO blood group and COVID-19: A review on behalf of the ISBT COVID-19 working group. Vox Sang. 2021, 116, 849-861. [CrossRef] [PubMed]

67. Le Pendu, J.; Breiman, A.; Deleers, M.; El Kenz, H.; Ruvoën, N. COVID-19 et groupes sanguins ABO-Où en est-on? Med. Sci. 2021, 37, 565-568. [CrossRef] 